Kragujevac Journal of Mathematics

Volume 42(1) (2018), Pages 7-14.

\title{
A REPRESENTATION FOR DERANGEMENT NUMBERS IN TERMS OF A TRIDIAGONAL DETERMINANT
}

\author{
FENG QI ${ }^{1,2,3}$, JING-LIN WANG ${ }^{3}$, AND BAI-NI GUO $^{4}$
}

\begin{abstract}
In the paper, the authors discover a representation for the derangement numbers in terms of a tridiagonal determinant. From the determinantal representation, the authors recover several identities and recurrence relations of the derangement numbers.
\end{abstract}

\section{INTRODUCTION}

In combinatorial mathematics, a derangement is a permutation of the elements of a set, such that no element appears in its original position. The number of derangements of a set of size $n$ is called the derangement number and usually denoted by $! n$. The subfactorial function is a map from $n$ to $! n$. The problem of counting derangements was first considered in 1708 and solved in 1713 by Pierre Raymond de Montmort, as did Nicholas Bernoulli at about the same time. The first eleven derangement numbers !n for $0 \leq n \leq 10$ are

$$
1, \quad 0, \quad 1, \quad 2, \quad 9, \quad 44, \quad 265, \quad 1854, \quad 14833, \quad 133496, \quad 1334961 .
$$

One of several expressions for computing the derangement numbers $n$ is

$$
! n=n ! \sum_{\ell=0}^{n} \frac{(-1)^{\ell}}{\ell !} \text {. }
$$

Key words and phrases. Derangement number, determinantal representation, tridiagonal determinant, generating function, identity, recurrence formula.

2010 Mathematics Subject Classification. Primary: 11B83. Secondary: 05A05, 05A10, 05A15, 11B37, 11B65, 11C20, 15A15.

Received: August 26, 2016.

Accepted: December 26, 2016. 
The derangement numbers $! n$ have an exponential generating function

$$
D(x)=\frac{e^{-x}}{1-x}=\sum_{n=0}^{\infty} ! n \frac{x^{n}}{n !} .
$$

The derangement numbers $! n$ arise naturally in many different contexts. More generally, the number of derangements in various families of transitive permutation groups has been studied extensively in recent years. For more and detailed information on the derangement numbers ! $n$, please refer to $[1,2,12,13]$ and plenty of references therein.

In [10], by studying the equation

$$
D(-x)=\frac{e^{x}}{1+x}=\sum_{n=0}^{\infty}(-1)^{n} ! n \frac{x^{n}}{n !},
$$

the authors corrected and recovered two determinantal representations for derangement numbers ! $n$. For more information, please refer to $[5,8]$ and closely related references therein.

The aim of this paper is, by computing the $n$th derivative of the exponential generating function $D(x)$, to find a representation for the derangement numbers $! n$ in terms of a tridiagonal determinant.

Our main result can be summarized up as the following theorem.

Theorem 1.1. For $n \in\{0\} \cup \mathbb{N}$, the derangement numbers $! n$ can be represented by a tridiagonal $(n+1) \times(n+1)$ determinant

$$
\begin{aligned}
& ! n=-\left|\begin{array}{ccccccccc}
-1 & 1 & 0 & 0 & 0 & \cdots & 0 & 0 & 0 \\
0 & 0 & 1 & 0 & 0 & \cdots & 0 & 0 & 0 \\
0 & -1 & 1 & 1 & 0 & \cdots & 0 & 0 & 0 \\
0 & 0 & -2 & 2 & 1 & \cdots & 0 & 0 & 0 \\
0 & 0 & 0 & -3 & 3 & \cdots & 0 & 0 & 0 \\
\vdots & \vdots & \vdots & \vdots & \vdots & \ddots & \vdots & \vdots & \vdots \\
0 & 0 & 0 & 0 & 0 & \cdots & n-3 & 1 & 0 \\
0 & 0 & 0 & 0 & 0 & \cdots & -(n-2) & n-2 & 1 \\
0 & 0 & 0 & 0 & 0 & \cdots & 0 & -(n-1) & n-1
\end{array}\right| \\
& =-\left|e_{i j}\right|_{(n+1) \times(n+1)} \text {, }
\end{aligned}
$$

where

$$
e_{i j}= \begin{cases}1, & i-j=-1 \\ i-2, & i-j=0 \\ 2-i, & i-j=1 \\ 0, & i-j \neq 0, \pm 1\end{cases}
$$

By virtue of the determinantal representation (1.4), we recover several identities and recurrence relations of the derangement numbers $! n$ in the form of remarks in the final section of this paper. 


\section{A LEMMA}

For supplying a concise proof for Theorem 1.1, we need the following lemma which was concluded in [6, Section 2.2, p. 849], [7, p. 94], [9, Remark 6], and [11, Lemma 2.1] from [3, p. 40, Exercise 5].

Lemma 2.1. Let $u(x)$ and $v(x) \neq 0$ be differentiable functions, let $U_{(n+1) \times 1}(x)$ be an $(n+1) \times 1$ matrix whose elements $u_{k, 1}(x)=u^{(k-1)}(x)$ for $1 \leq k \leq n+1$, let $V_{(n+1) \times n}(x)$ be an $(n+1) \times n$ matrix whose elements

$$
v_{i, j}(x)= \begin{cases}\left(\begin{array}{l}
i-1 \\
j-1
\end{array}\right) v^{(i-j)}(x), & i-j \geq 0 \\
0, & i-j<0\end{cases}
$$

for $1 \leq i \leq n+1$ and $1 \leq j \leq n$, and let $\left|W_{(n+1) \times(n+1)}(x)\right|$ denote the determinant of the $(n+1) \times(n+1)$ matrix

$$
W_{(n+1) \times(n+1)}(x)=\left[U_{(n+1) \times 1}(x) \quad V_{(n+1) \times n}(x)\right] .
$$

Then the nth derivative of the ratio $\frac{u(x)}{v(x)}$ can be computed by

$$
\frac{\mathrm{d}^{n}}{\mathrm{~d} x^{n}}\left[\frac{u(x)}{v(x)}\right]=(-1)^{n} \frac{\left|W_{(n+1) \times(n+1)}(x)\right|}{v^{n+1}(x)} .
$$

\section{Proofs of Theorem 1.1}

Now we are in a position to provide a concise proof for Theorem 1.1.

Applying $u(x)=e^{-x}$ and $v(x)=1-x$ in Lemma 2.1 gives

$$
u_{k, 1}=\left(e^{-x}\right)^{(k-1)}=(-1)^{k-1} e^{-x} \rightarrow(-1)^{k-1},
$$

for $1 \leq k \leq n+1$ as $x \rightarrow 0$ and

$$
\begin{gathered}
v_{i, j}=\left(\begin{array}{l}
i-1 \\
j-1
\end{array}\right)(1-x)^{(i-j)}= \begin{cases}\left(\begin{array}{l}
i-1 \\
j-1
\end{array}\right)(1-x), & i-j=0, \\
-\left(\begin{array}{l}
i-1 \\
j-1
\end{array}\right), & i-j=1, \\
0, & i-j \neq 0,1,\end{cases} \\
=\left\{\begin{array} { l l } 
{ 1 - x , } & { i - j = 0 , } \\
{ 1 - i , } & { i - j = 1 , } \\
{ 0 , } & { i - j \neq 0 , 1 , }
\end{array} \rightarrow \left\{\begin{array}{ll}
1, & i-j=0, \\
1-i, & i-j=1, \\
0, & i-j \neq 0,1,
\end{array}\right.\right.
\end{gathered}
$$


for $1 \leq i \leq n+1$ and $1 \leq j \leq n$ as $x \rightarrow 0$. Consequently, by virtue of the formula (2.1), we have

$$
\begin{gathered}
\frac{\mathrm{d}^{n} D(x)}{\mathrm{d} x^{n}}=\frac{(-1)^{n}}{(1-x)^{n+1}}\left|\begin{array}{ccccccc}
e^{-x} & 1-x & 0 & \cdots & 0 & 0 \\
-e^{-x} & -1 & 1-x & \cdots & 0 & 0 \\
e^{-x} & 0 & -2 & \cdots & 0 & 0 \\
\vdots & \vdots & \vdots & \ddots & \vdots & \vdots \\
(-1)^{n-2} e^{-x} & 0 & 0 & \cdots & 1-x & 0 \\
(-1)^{n-1} e^{-x} & 0 & 0 & \cdots & -(n-1) & 1-x \\
(-1)^{n} e^{-x} & 0 & 0 & \cdots & 0 & -n
\end{array}\right| \\
\begin{array}{ccccccc}
1 \\
-1 & -1 & 0 & 0 & \cdots & 0 & 0 \\
1 & 0 & -2 & 1 & \cdots & 0 & 0 \\
-1 & 0 & 0 & -3 & \cdots & 0 & 0 \\
\vdots & \vdots & \vdots & \vdots & \ddots & \vdots & \vdots \\
(-1)^{n-2} & 0 & 0 & 0 & \cdots & 1 & 0 \\
(-1)^{n-1} & 0 & 0 & 0 & \cdots & -(n-1) & 1 \\
(-1)^{n} & 0 & 0 & 0 & \cdots & 0 & -n
\end{array} \mid
\end{gathered}
$$

as $x \rightarrow 0$ for $n \geq 0$. Therefore, since $D(x)$ is a generating function of $! n$, as showed in (1.3), we obtain

$$
! n=\lim _{x \rightarrow 0} \frac{\mathrm{d}^{n} D(x)}{\mathrm{d} x^{n}}=(-1)^{n}\left|\begin{array}{ccccccc}
1 & 1 & 0 & 0 & \cdots & 0 & 0 \\
-1 & -1 & 1 & 0 & \cdots & 0 & 0 \\
1 & 0 & -2 & 1 & \cdots & 0 & 0 \\
-1 & 0 & 0 & -3 & \cdots & 0 & 0 \\
\vdots & \vdots & \vdots & \vdots & \ddots & \vdots & \vdots \\
(-1)^{n-2} & 0 & 0 & 0 & \cdots & 1 & 0 \\
(-1)^{n-1} & 0 & 0 & 0 & \cdots & -(n-1) & 1 \\
(-1)^{n} & 0 & 0 & 0 & \cdots & 0 & -n
\end{array}\right| .
$$

Adding the $n$th row to the $(n+1)$ th row, then the $(n-1)$ th row to the $n$th row,$\ldots$, then the 1st row to the 2 nd row of the above determinant yield

$$
! n=(-1)^{n}\left|\begin{array}{ccccccc}
1 & 1 & 0 & 0 & \cdots & 0 & 0 \\
0 & 0 & 1 & 0 & \cdots & 0 & 0 \\
0 & -1 & -1 & 1 & \cdots & 0 & 0 \\
0 & 0 & -2 & -2 & \cdots & 0 & 0 \\
\vdots & \vdots & \vdots & \vdots & \ddots & \vdots & \vdots \\
0 & 0 & 0 & 0 & \cdots & 1 & 0 \\
0 & 0 & 0 & 0 & \cdots & 2-n & 1 \\
0 & 0 & 0 & 0 & \cdots & 1-n & 1-n
\end{array}\right|_{(n+1) \times(n+1)}
$$

Using -1 to multiply all even rows and all odd columns of the above determinant immediately results in (1.4). The proof of Theorem 1.1 is complete. 


\section{REMARKS}

After supplying a concise proof for Theorem 1.1, we show the significance of the determinantal representation (1.4) by listing several remarks below.

Remark 4.1. By expanding the determinant in (3.1) according to the first row or the first column consecutively, we can recover the expression (1.2).

Remark 4.2. By expanding the determinant in (3.1) according to the $n$th row or according to the $n$th column, we can easily recover the recurrence relation

$$
! n=(-1)^{n}+n \times !(n-1), \quad n \in \mathbb{N} .
$$

Remark 4.3. By expanding the determinant in (1.4) according to the $n$th row or according to the $n$th column, we can recover the recurrence relation

$$
! n=(n-1)[!(n-1)+!(n-2)], \quad n \geq 2 .
$$

Remark 4.4. By expanding the determinant in (1.4) according to the first row or according to the first column, we can obtain

$$
! n=\left|\begin{array}{cccccc}
0 & 1 & 0 & \cdots & 0 & 0 \\
-1 & 1 & 1 & \cdots & 0 & 0 \\
0 & -2 & 2 & \cdots & 0 & 0 \\
\vdots & \vdots & \vdots & \ddots & \vdots & \vdots \\
0 & 0 & 0 & \cdots & 1 & 0 \\
0 & 0 & 0 & \cdots & n-2 & 1 \\
0 & 0 & 0 & \cdots & -(n-1) & n-1
\end{array}\right|_{n \times n} \quad, \quad n \in \mathbb{N}
$$

Further expanding the above determinant according to the first row or according to the first column, we can obtain

$$
! n=\left|\begin{array}{ccccccc}
2 & 1 & 0 & \cdots & 0 & 0 & 0 \\
-3 & 3 & 1 & \cdots & 0 & 0 & 0 \\
0 & -4 & 4 & \cdots & 0 & 0 & 0 \\
\vdots & \vdots & \vdots & \ddots & \vdots & \vdots & \vdots \\
0 & 0 & 0 & \cdots & n-3 & 1 & 0 \\
0 & 0 & 0 & \cdots & -(n-2) & n-2 & 1 \\
0 & 0 & 0 & \cdots & 0 & -(n-1) & n-1
\end{array}\right|_{(n-2) \times(n-2)} \quad, \quad n \geq 3
$$


Remark 4.5. In general, for all integers $k$ with $k \leq n-1$, define the tridiagonal determinant

$$
D_{n}(k)=\left|\begin{array}{ccccccc}
k & 1 & 0 & \cdots & 0 & 0 & 0 \\
-(k+1) & k+1 & 1 & \cdots & 0 & 0 & 0 \\
0 & -(k+1) & k+2 & \cdots & 0 & 0 & 0 \\
\vdots & \vdots & \vdots & \ddots & \vdots & \vdots & \vdots \\
0 & 0 & 0 & \cdots & n-3 & 1 & 0 \\
0 & 0 & 0 & \cdots & -(n-2) & n-2 & 1 \\
0 & 0 & 0 & \cdots & 0 & -(n-1) & n-1
\end{array}\right|
$$

It is clear that $D_{n}(-1)=-! n$ for $n \geq 0, D_{n}(0)=! n$ for $n \geq 1$, and $D_{n}(2)=! n$ for $n \geq 3$. Moreover, the determinant $D_{n}(k)$ satisfies

$$
\begin{aligned}
& D_{n}(n-1)=n-1, \\
& D_{n}(n-2)=\left|\begin{array}{cc}
n-2 & 1 \\
-(n-1) & n-1
\end{array}\right|=(n-1)^{2} \text {, } \\
& D_{n}(n-3)=\left|\begin{array}{ccc}
n-3 & 1 & 0 \\
-(n-2) & n-2 & 1 \\
0 & -(n-1) & n-1
\end{array}\right|=(n-1)\left(n^{2}-3 n+1\right) \text {, } \\
& D_{n}(n-4)=\left|\begin{array}{cccc}
n-4 & 1 & 0 & 0 \\
3-n & n-3 & 1 & 0 \\
0 & 2-n & n-2 & 1 \\
0 & 0 & 1-n & n-1
\end{array}\right|=(n-1)\left(n^{3}-6 n^{2}+9 n-1\right)
\end{aligned}
$$

and the recurrence relation

$$
D_{n}(k)=k D_{n}(k+1)+(k+1) D_{n}(k+2), \quad k \leq n-1 .
$$

Remark 4.6. Directly combining (1.2) with (4.1) arrives at

$$
\left|\begin{array}{ccccccc}
2 & 1 & 0 & \cdots & 0 & 0 & 0 \\
-3 & 3 & 1 & \cdots & 0 & 0 & 0 \\
0 & -4 & 4 & \cdots & 0 & 0 & 0 \\
\vdots & \vdots & \vdots & \ddots & \vdots & \vdots & \vdots \\
0 & 0 & 0 & \cdots & n-3 & 1 & 0 \\
0 & 0 & 0 & \cdots & -(n-2) & n-2 & 1 \\
0 & 0 & 0 & \cdots & 0 & -(n-1) & n-1
\end{array}\right|_{(n-2) \times(n-2)}=n ! \sum_{\ell=0}^{n} \frac{(-1)^{\ell}}{\ell !}
$$

for $n \geq 3$. 
Remark 4.7. From the first proof of Theorem 1.1, we can conclude that the $n$th derivative of the generating function $D(x)$ can be computed by

$$
\begin{aligned}
\frac{\mathrm{d}^{n} D(x)}{\mathrm{d} x^{n}}= & \frac{(-1)^{n} e^{-x}}{(1-x)^{n+1}}\left|\begin{array}{cccccc}
-x & 1-x & 0 & \cdots & 0 & 0 \\
-1 & -1-x & 1-x & \cdots & 0 & 0 \\
0 & -2 & -2-x & \cdots & 0 & 0 \\
\vdots & \vdots & \vdots & \ddots & \vdots & \vdots \\
0 & 0 & 0 & \cdots & 1-x & 0 \\
0 & 0 & 0 & \cdots & 2-n-x & 1-x \\
0 & 0 & 0 & \cdots & 1-n & 1-n-x
\end{array}\right| \\
& =\frac{(-1)^{n} e^{-x}}{(1-x)^{n+1}}\left|e_{i j}(x)\right|_{n \times n},
\end{aligned}
$$

where $n \in \mathbb{N}$ and

$$
e_{i j}(x)= \begin{cases}1-x, & i-j=-1, \\ 1-i-x, & i-j=0, \\ 1-i, & i-j=1, \\ 0, & i-j \neq 0, \pm 1 .\end{cases}
$$

Remark 4.8. In [4], it was deduced that

$$
!(r+1)=\left|\begin{array}{ccccccc}
1 & 1 & 0 & 0 & \cdots & 0 & 0 \\
-1 & 1 & 2 & 0 & \cdots & 0 & 0 \\
0 & -1 & 2 & 3 & \cdots & 0 & 0 \\
0 & 0 & -1 & 3 & \cdots & 0 & 0 \\
\vdots & \vdots & \vdots & \vdots & \ddots & \vdots & \vdots \\
0 & 0 & 0 & 0 & \cdots & r-1 & r \\
0 & 0 & 0 & 0 & \cdots & -1 & r
\end{array}\right|,
$$

for $r \in \mathbb{N}$. By this determinantal expression for the derangement numbers ! $(r+1)$, we figure out that $! 2=1, ! 3=2, ! 4=6$, and $! 5=24$. But, the latter two values do not coincide with their corresponding ones in (1.1). This means that the expression (4.2) is wrong.

\section{REFERENCES}

[1] M. Aigner, A Course in Enumeration, Graduate Texts in Mathematics, 238, Springer, Berlin, 2007.

[2] T. Andreescu and Z. Feng, A Path to Combinatorics for Undergraduates-Counting Strtegies, Birkhäuser, Boston-Basel-Berlin, 2004.

[3] N. Bourbaki, Elements of Mathematics: Functions of a Real Variable: Elementary Theory, Translated from the 1976 French original by Philip Spain. Elements of Mathematics. SpringerVerlag, Berlin, 2004.

[4] M. Janjić, Determinants and recurrence sequences, J. Integer Seq. 15 (3) (2012) Article ID 12.3.5.

[5] F. Qi, A determinantal representation for derangement numbers, Glob. J. Math. Anal. 4 (3) (2016), 17-17. 
[6] F. Qi, Derivatives of tangent function and tangent numbers, Appl. Math. Comput. 268 (2015), 844-858.

[7] F. Qi and R. Chapman, Two closed forms for the Bernoulli polynomials, J. Number Theory 159 (2016), 89-100.

[8] F. Qi and B.-N. Guo, Explicit formulas for derangement numbers and their generating function, J. Nonlinear Funct. Anal. 2016, Article ID 45.

[9] F. Qi, X.-T. Shi and F.-F. Liu, Several identities involving the falling and rising factorials and the Cauchy, Lah, and Stirling numbers, Acta Univ. Sapientiae Math. 8 (2) (2016), 282-297.

[10] F. Qi, J.-L. Wang and B.-N. Guo, A recovery of two determinantal representations for derangement numbers, Cogent Math. 3 (2016), Article ID 1232878.

[11] C.-F. Wei and F. Qi, Several closed expressions for the Euler numbers, J. Inequal. Appl. 2015 (2015), Article ID 219.

[12] H. S. Wilf, Generatingfunctionology, Second edition, Academic Press Inc., Boston, MA, 1994.

[13] H. S. Wilf, Generatingfunctionology, Third edition. A. K. Peters Ltd., Wellesley, MA, 2006.

\author{
${ }^{1}$ Institute of Mathematics \\ Henan Polytechnic University \\ JiAOZUO City, 454010 \\ Henan Province \\ China \\ ${ }^{2}$ College of Mathematics \\ InNer Mongolia University for Nationalities \\ Tongliao City, 028043 \\ Inner Mongolia Autonomous Region \\ CHINA \\ E-mail address: qifeng618@gmail.com \\ ${ }^{3}$ Department of Mathematics \\ College of SCIEnCE \\ Tianjin Polytechnic University \\ Tianjin City, 300387 \\ ChIna \\ E-mail address: jing-lin.wang@hotmail.com \\ ${ }^{4}$ SCHOOL OF MATHEMATICS AND INFORMATICS \\ Henan Polytechnic University \\ JiAOZUO City, 454010 \\ Henan Province \\ ChInA \\ E-mail address: bai.ni.guo@hotmail.com
}

